\title{
A Methodology For Excitation Systems Identification
}

\author{
Héctor Antonio Botero Castro ${ }^{1}$, José Miguel Ramírez Scarpetta, IEEE Member ${ }^{2}$ \\ ${ }^{1}$ Dept. of Electrical Eng, Universidad Nacional de Colombia, Facultad de Minas. Grupo de Automática Universidad \\ Nacional de Colombia (Gaunal), email : habotero@unalmed.edu.co \\ ${ }^{2}$ Shool. of Electrical and Electronics Eng, Universidad del Valle, Grupo de Investigación en Control Industrial \\ (Gici), email jomiram@univalle.edu.co
}

\begin{abstract}
In this paper a methodology for parameter identification of the IEEE standard 421.5 (1992) excitation systems models is presented. The methodology applies parametric identification techniques to the linear dynamics of voltage regulator models. A pseudorandom binary sequence small signal is used in an offline generator test, that allows a reduction in the equipment test time, in comparison with step or frequency response methods. The remaining parameters for the saturation nonlinearities and exciter magnetic saturation are obtained by means of large signal excursion simple tests. The methodology is illustrated applying it to the IEEE ST1A model in simulation, and experimentally to a direct digital auto excited excitation system, represented with the IEEE ST4B model. Simulation and experimental results show the validity of the proposed methodology, with achieved successes percentages of $96 \%$ for simulation and $73 \%$ for experimentation.
\end{abstract}

\section{INTRODUCTION}

Modern power systems are constantly subjected to disturbances such as sudden load and generation changes, equipment flaws, transmission lines and distribution network reconfiguration, atmospheric disturbances and attacks with explosives.

Additionally, economy globalisation and treaties among neighbouring countries have encouraged electric systems interconnection.

Due to this, systems are now subjected to additional disturbances. It also has pushed towards a strong tendency to take equipment to its operative limits.

Therefore, excitation systems modelling and identification gains importance because power systems analysis results reliability greatly depends upon models accuracy.

Aiming to obtain appropriate models, several investigators around the world have studied and applied methods for parameter identification of excitation systems:

In [4] reports a frequency response method for identifying parameters in an electromechanical regulator. In [7] apply a method with model order reduction using a technique based on the Modes of Energy.

In [5] employ the general minimum squares method for a ST excitation system.

In [16] use a stochastic approach method to determine the DC1 and AC1 excitation systems parameters.

The sensibility matrix method for identifying excitation systems is treated in [1] and [14].
In [9] the author proposes an identification methods based on an autoregressive model to determine the parameters of a AC1 excitation system.

The mentioned methods have inconveniences since they are mathematically complex and use techniques not common to the electric generation industry. Additionally the documents that report the results are not sufficiently explanatory so that non expert personal can use them in a simple manner. Except by [3] who describes a friendly and easy to use program for the synchronous generator and the excitation system parameters identification, authors do not explain the computational aids necessary to solve the resulting equations and assume that the reader has enough expertise to carry out the task.

In some articles only few parameters are considered while other are left fixed or are supposed known. This reduces model accuracy because the fixed parameters are based on typical model parameters, which usually has very wide variation ranges.

Additionally, some authors use discrete models based on numeric approaches as the bilinear transformation. These approaches add constraints to the parameters search process, because it is not easy to interpret the results in the $\mathrm{Z}$ domain with parameters that don't match with the real system physical elements such as gains, time constants and signal limits.

Based in the previous problem, and aiming to propose a tool allowing to obtain the excitation systems parameters in a simple fashion, in this article the authors present a methodology applied to two excitation systems types: ST1A and ST4B of [6] that are commonly found in power systems.

The methodology can be studied and applied by engineers with some knowledge of power systems and modelling of dynamic systems.

\section{PROPOSED METHODOLOGY}

The proposed procedure is based on two approaches: - Tests methodology performed in the Salvajina power station excitation control system reported in [11] and [12]. The methodology is based on the determination of gains, time constants, limits and additional parameters based on the model structure, the model behaviour governing differential equations and the previously measured signals. This approach is close to what is known as "white box approach". In general small and large signal tests are used, the latter to determine the limits of the signals involved in the model. 
- Parametric identification methodology for linear systems with predetermined model's structure, by means of which a system model based on international standards is proposed.

With the proposed model, a linear representation of the dynamic system in state space is obtained, the parameters to be identified are selected and the model parameters are obtained through the application of the systems identification technique.

The methodology is as follows:

\subsection{System mathematical model attain}

Initially a structure is selected for the mathematical model. This structure can be obtained from the norm [6].

\subsection{Nonlinearities definition and measure}

The main nonlinearities of the excitation control systems are located in the magnetic saturation of the exciter and the synchronous generator and the physical limits imposed in some components output signals. The identification of saturation function is carried out measuring the exciter and generator input and output in two operation points, without and with saturation [6].

For the identification of limits type nonlinearities, either great magnitude signals can be applied in the regulators electronic cards or periodic signal of high amplitude and high frequency that saturate transitorily the variables of interest [11].

\subsection{Model Linearization around the operation point}

The excitation systems models should be linearized because the identification methods to be applied suppose that the system is linear.

The limits can be eliminated if it is considered that changes in the system don't have appreciable magnitudes that overcome them. The saturation function is differentiable and can be linearized around an operation point.

By means of the linearization it is possible to obtain the standard state variables representation for linear invariant system:

$$
\begin{aligned}
& \frac{d X}{d t}=A^{*} X+B^{*} U \\
& Y=C X+D U
\end{aligned}
$$

Carrying out an analysis over the matrix $A$ it can be observed that the parameters $K_{A}, T_{A}, K_{F}, T_{F}$ can be obtained independently by means of a linear identification technique, where:

$K_{A}:$ Regulator gain

$K_{F}:$ Stabilization network gain

$T_{A}$ : Regulator time constant

$T_{F}$ : Stabilization time constant

To complete the model parameters, the constants related to the regulation of thyristor bridge, the measure filter, and field constants of the synchronous generator can be measured using standard tests.

\subsection{System general dynamics attain}

An important step in the identification process is to set and perform an experiment to gather information on the dynamic of the system. To do so, it is necessary to carry out a step test in the real system to know its settling time $T_{S}$. This test is done generating a step change in the reference voltage and recording the dynamics of synchronous generator no load output voltage. The step magnitude should be such that the system nonlinearities don't get excited.

\subsection{Input signal calculation}

The parametric identification methods demand that the applied input signal be of persistent excitation in order to [15]:

- Obtain a consistent and unbiased estimate and to avoid singular solutions,

- Obtain a permanent variation but with a very low correlation,

- Excite the system important dynamics in the frequencies range of interest for the model application.

To complete the above-mentioned, a Pseudo Random Binary Sequence is used (PRBS). The PRBS is a two levels signal composed of a succession of pulses modulated in its width, so that it approaches a white noise with a rich content in frequencies. The PRBS is periodic, deterministic and can be generated by means of a shift register with sum in module 2 [15].

The PRBS is characterised by three parameters:

- The signal level, which determines its maximum and minimum value.

- The number of bits of the shift register $n_{r}$, which determines the signal longitude: $N=2^{n_{r}-1}$

- The clock period $T_{s w}$, it modifies the signal duration and its spectral density power.

$n_{r}$ and $T_{s w}$ selection can be done considering that the power spectrum of the PRBS is determined by ([15] and [8] ) :

$$
\phi_{U}(w)=\frac{a^{2}(N+1) T_{s w}}{N}\left[\frac{\operatorname{sen}\left(0.5 w T_{s w}\right)}{0.5 w T_{s w}}\right]^{2}
$$

where:

$N$ : PRBS longitude

$w:$ Frequency $(\mathrm{rad} / \mathrm{s})$

The band width of the power spectrum is reached at the value:

$$
w_{B}=\frac{2.8}{T_{s w}}
$$

In this frequency the signal reaches half of the power spectrum density maximum value. Due to this and to the PRBS covariance function periodicity, the useful range of this signal is between:

$$
\frac{2 \pi}{N T_{s w}} \leq w \leq \frac{2.8}{T_{s w}}\left(\frac{r a d}{s}\right)
$$


Now, the widest pulse in the PRBS duration is $n_{r} T_{s w}$ and the duration of this pulse should be approximately equal to the system's settling time $T_{S}$, in order to correctly determine the static system's gain. The procedure to calculate the PRBS is:

- Determine the system's settling time $T_{S}$.

- Estimate the frequencies band of interest for the system.

- Consider $n_{r}$ fixed (for example $n_{r}=8$ ) and calculate the $T_{s w}$ as:

$T_{s w}=\frac{1.1 T_{S}}{n_{r}}$

- Verify that the frequencies band of interest is covered with the selection of $T_{s w}$, use equation (5) for it.

- Verify that the sampling time $T$ is multiple of $T_{s w}$. ( $T=\frac{T_{s w}}{4}$ is recommended).

- Verify that all the criteria is met, otherwise change $n_{r}$ and/or $T_{s w}$ and repeat.

For the PRBS magnitude determination two criteria explained before should be applied:

- The magnitude should be such that the nonlinearities such as limits and physical restrictions do not get excited, neither risk equipment operation.

- The signal-noise relationship should be high. This can be verified observing the data.

Some considerations to be kept in mind when selecting the sampling time $T$ are [8]:

- The sampling time $T$ is selected considering a commitment between noise reduction and capture of the system fast dynamics.

- If the sampling time is very small, numeric problems can arise in the algorithms due to the introduced noise.

- If the sampling time is very big compared with the system time constants, variance errors may appear in the identification.

- The excitation system lowest time constant $\left(T_{R}\right)$ is located in the terminal voltages measure circuit filter. This time constant has a typical value of $20 \mathrm{~ms}$, which suggests a sampling time of minimum $10 \mathrm{~ms}$ so that 10 samples are captured in this filter dynamic response, avoiding to lose information in the data acquisition process.

- In some practical experiences such as [2] and others the sampling time was of $1 \mathrm{~ms}$. Several companies offer equipment for Data Acquisition that fulfil the requirements for sampling at these speeds.

\subsection{The predictor error method}

Parameters related to the linear dynamics are found by means of the Predictor Error Method after having the input and output data. Using this method, parameters estimates and their deviations are found. Additionally, it can be found the error in the identification process.
By means of the predictor error method one intends to determine the vector of parameters $\theta$, such that the prediction error:

$$
\varepsilon(t, \theta)=y(t)-\hat{y}\left(\left.t\right|_{t-1}, \theta\right)
$$

be small, where:

\section{$\varepsilon(t, \theta):$ Prediction error}

$y(t)$ : Output measure

$\hat{y}\left(\left.t\right|_{t-1}, \theta\right)$ :Prediction of $y(t)$ with past values

$\theta$ : parameters vector to be estimate

To apply the method the system is considered to be able to take the general model structure form:

$$
y(t)=G\left(q^{-1}, \theta\right) u(t)+H\left(q^{-1}, \theta\right) e(t)
$$

where:

$u(t):$ Input

$e(t)$ : sequence of independent random variables with zero mean (White noise).

$G\left(q^{-1}, \theta\right), H\left(q^{-1}, \theta\right)$ : finite dimension filters

$q^{-1}$ : shift operator

and that a linear predictor can be build of the form:

$\hat{y}\left(\left.t\right|_{t-1}, \theta\right)=L_{1}\left(q^{-1}, \theta\right) y(t)+L_{2}\left(q^{-1}, \theta\right) u(t)$

calculated with past data and predictor filters: $L_{1}\left(q^{-1}, \theta\right)$ and $L_{2}\left(q^{-1}, \theta\right)$.

From equation (9), the predictor can be built in several ways for the model (8). Given the model and predictor, the prediction error is calculated with (7) for all data. Finally the estimated value $\theta$ is obtained such that an error criterion like:

$R_{N}(\theta)=\frac{1}{N} \sum_{t=1}^{N} \varepsilon(t, \theta) \varepsilon^{T}(t, \theta)$

is minimized. The process is illustrated graphically in Figure 1.

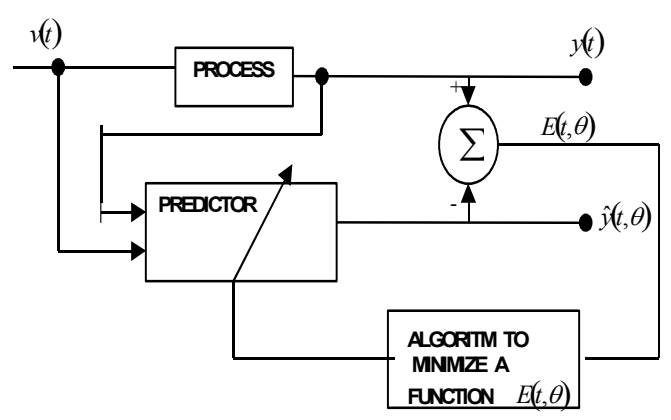

Fig 1. Process for Identification 


\subsection{Tool for identification}

The identification process is simplified using a tool implemented in Matlab by means of the System Identification Toolbox. The tool has the following programmed functions useful to carry out the following operations sequence:

- Loads data file, that is to say the data recorded in an oscilloscope.

- Organises and filters data, removing mean values and noise. Additionally it separates data in two sets, for identification and validation.

- Applies predictor error method, by means of a state variables system model.

- Carries out validation process, comparing results graphically. Additionally presents the hits percentage and the correlation analysis of the residuals with the input.

\section{SIMULATION RESULTS}

The identification methodology was tested simulating a static excitation system whose parameters were measured in a real plant model [12] and [13]. The system is represented by means the ST1A model of the norm [6]. The data were obtained from simulations carried out in Matlab - Simulink. In the identification process two computer programs were used: one to contain the parameters and one to generate the data.

Data for the identification process are obtained from the simulation of the excitation control system. The simulator incorporates functions that allow verification of the system identification with Pseudo Random Binary Sequence (PRBS) and considers two types of noise signals in the measure: Random noise (white) and $360 \mathrm{~Hz}$ noise present in the thyristor bridge. The simulator is implemented so that the PRBS signal can be applied after system establishment in an operation point.
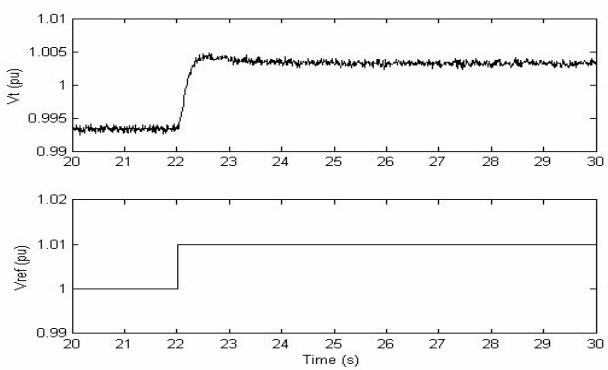

Fig 2. ST1A system step response

This operation point can be adjusted from the voltage reference. Input and output data simulation result are attained in two formats: graphical forms and data in Matlab with extension. mat. The sampling time was taken as $10 \mathrm{~ms}$ to perform a conservative approach. Results are shown in Figures 2 to 4 .

Additionally the residuals correlation function and the correlation between the residuals and the input are shown in Figure 5. The demarcation lines indicate the confidence interval functions. It is desirable that no point leaves these fringes, however residuals correlation is very dependent in input type and therefore is not considered decisive in the validation.
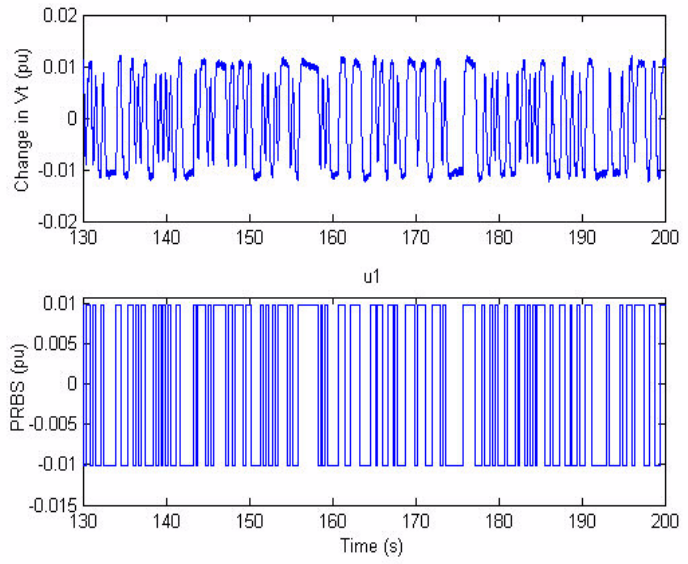

Fig 3. ST1A registered data.

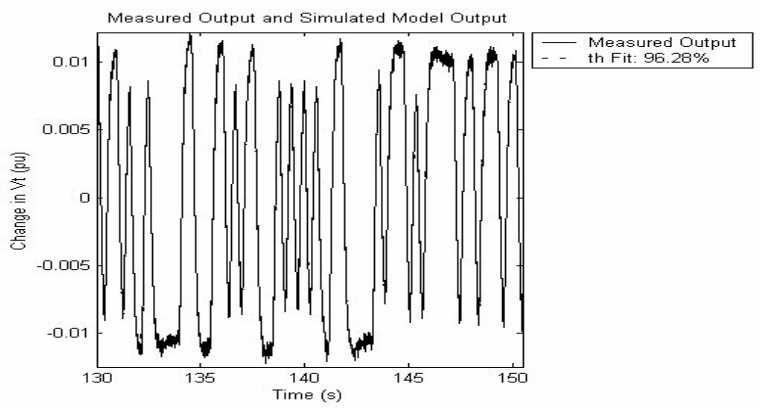

Fig 4. Comparison with ST1A validation data
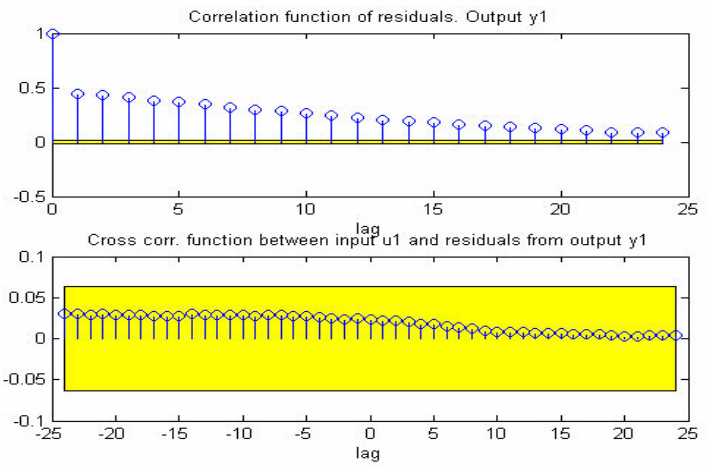

Fig 5. ST1A correlation functions

Finally the parameters and their typical deviation are found, see Table 1 for this case.

An additional validation is done by comparing the field voltage measured and simulated signals. The parameters used by the simulator are those calculated in the identification process. Simulator input is the same measured input. This comparison is important because the field voltage dynamics has fast variation. Comparison is shown in Figure 6.

Results in this case are very good; there is a good coincidence when comparing the graphics, additionally successes percentage is high and therefore the identified parameters are accepted. Since the measured parameters are known, it is possible to verify that the identified parameters deviations from the true parameters are very small. 


\begin{tabular}{|l|l|l|l|}
\hline Parameter & $\begin{array}{l}\text { Identified } \\
\text { value and } \\
\text { deviation }\end{array}$ & $\begin{array}{l}\text { Theoretic } \\
\text { real value }\end{array}$ & $\begin{array}{l}\text { Error } \\
\text { Percentage }\end{array}$ \\
\hline$K_{A}$ (p.u) & $\begin{array}{l}167.6927 \\
(+/-) 2.8726\end{array}$ & 165.15 & $1.53 \%$ \\
\hline$T_{A}$ (p.u) & $\begin{array}{l}0.1620 \\
(+/-) 0.0028\end{array}$ & 0.16 & $1.25 \%$ \\
\hline $\begin{array}{l}K_{F} \\
\text { (p.u) }\end{array}$ & $\begin{array}{l}0.0177 \\
(+/-) 0.0002\end{array}$ & 0.0175 & $1.14 \%$ \\
\hline $\begin{array}{l}T_{F} \\
\text { (p.u) }\end{array}$ & $\begin{array}{l}0.8643 \\
(+/-) 0.0058\end{array}$ & 0.86 & $0.5 \%$ \\
\hline
\end{tabular}

Table 1. ST1A system identified parameters

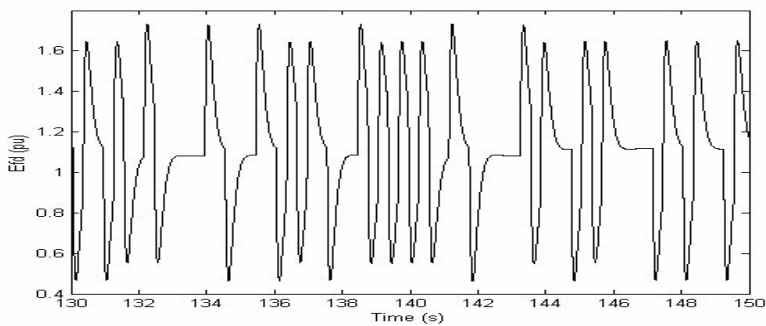

Fig 6. ST1A field voltage signal comparison (Solid: Identified Parameters, Dot: Model Parameters)

Field signals comparison allows to analyze the model capacity to generate intermediate signals. In this case signals coincide almost exactly

\section{REAL CASE ATTAINED RESULTS}

Aiming to verify the methodology in a real case, tests were performed over an excitation system built at the Universidad del Valle [10]. The system contains the following elements:

- A 3 KVA, 8 A, 208 V, delta connection, P.F. 0.7-1, 1800 rpm synchronous generator.

- A motor load consisting of a $216 \mathrm{~V}$, delta connection, 15.5 A, $5 \mathrm{Kw}$, P.F. $=1$ synchronous motor.

- An actuator or exciter consisting of a completely controlled bridge.

- A voltage regulator implemented in a Texas Instrument DSP (Digital Signal Processing) TMS 320C6711 series. The control algorithm was programmed in this processor using $\mathrm{C}$ language to implement the difference equations obtained in the design.

Aiming to gain an idea of the system general dynamics an experiment was carried out in order to obtain the open loop step change response; by a change in the shot angle from the DSP in open loop, a step in the field voltage was obtained. This excitation variation produces a change in the terminals voltage as shown in Figure 7. The voltage step represents a $10 \%$ variation of the nominal voltage.

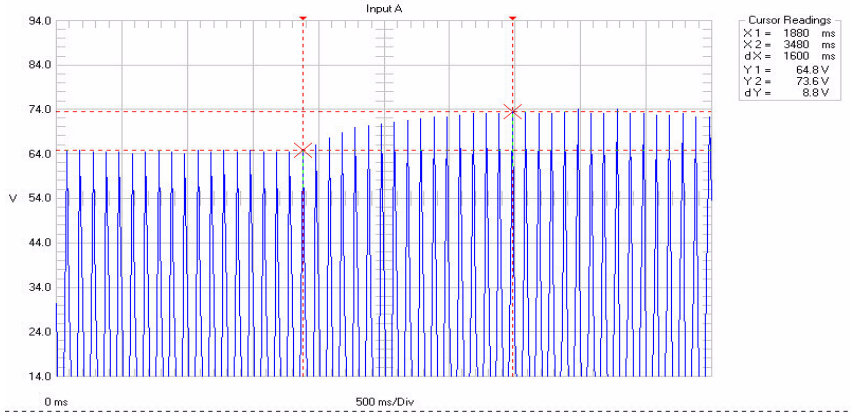

Fig 7. ST4B system step response

\section{Linear discretized Model}

The excitation system model's block diagram is shown in Figure 8; is a ST4B type model neglecting the actuator dynamics $\left(T_{A}=0\right)$; the thyristor bridge regulation is considered by the $K_{c}$ constant. It is assumed $K_{A}=1$ since excitation system gain is considered by the constant $K_{p}$.

Results of identification process are shown in Figures 9 11 and Table 2.

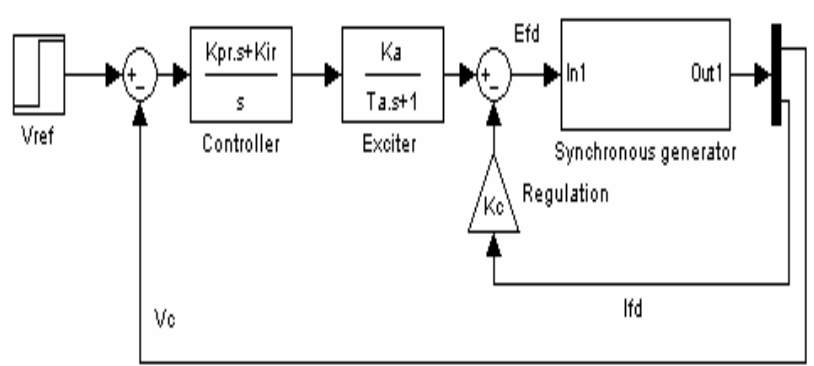

Fig. 8. ST4B Simplified Model
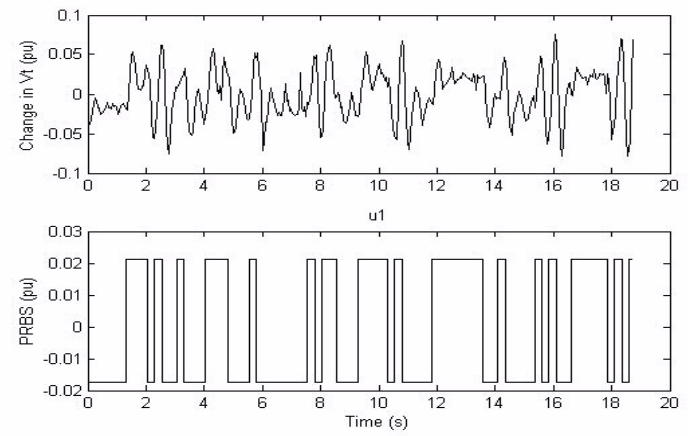

Fig. 9. ST4B system registered data

Results in this case are good; the model was not validated with the field voltage because this signal was not measured. 


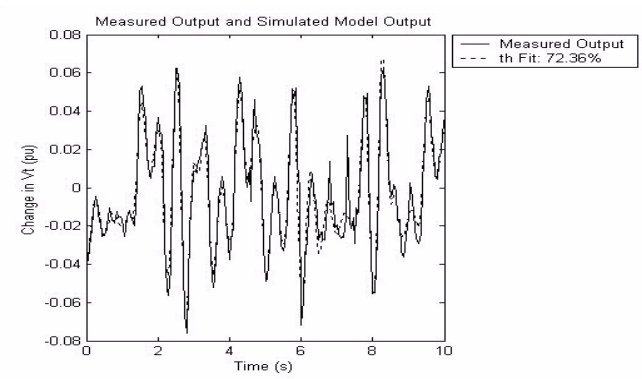

Fig. 10. Comparison with validation data
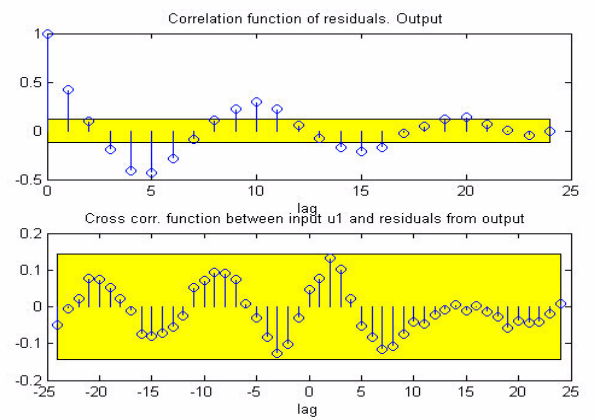

Fig. 11. ST4B correlation functions

\begin{tabular}{|l|l|}
\hline Parameter & $\begin{array}{l}\text { Identified } \\
\text { value and its } \\
\text { deviation }\end{array}$ \\
\hline$K_{P R}$ (p.u) & $\begin{array}{l}0.2540 \\
(+/-) 0.0716\end{array}$ \\
\hline$K_{I R}$ (p.u) & $\begin{array}{l}73.8923 \\
(+/-) 0.9625\end{array}$ \\
\hline
\end{tabular}

Table 2. ST4B identified system parameters

\section{CONCLUSIONS}

The methodology was applied to excitation systems detailed models proposed in IEEE Std 421.5. 1992. Attempting to obtain conditions close to reality, other interference signals present in real systems were added to the models, such that measure noise and noise introduced by controlled rectifiers circuits.

An advantage of the method is that only one input signal added to the reference signal is applied, which can be introduced in the control algorithm if the system is digital or by means of the programmed commutation of a resistance if the system is analogue.

The measures were only performed in the input and output signals, that is to say in the reference and in the synchronous generator voltage transducer in terminals. Other methods described in the literature apply partial signals to identify each component dynamics. This last technique has the disadvantage of feedback, because the refereed signals have a high correlation component which generates error in the identification procedure.

The methodology was applied to a real system and its parameters were identified. The real plant tests data have the PRBS signal as input. This signal has a high persistence order which eases its use in parametric identification methods.

The proposed methodology is useful as a base for a excitation systems parameters identification study. The programs implemented in Matlab contain the necessary and recommended functions to carry out the identification process. The use of Matlab as calculation tool simplifies the mathematical handling and speeds up the identification, if considered that this program is of daily handling in University and Industrial environments.

\section{REFERENCES}

[1] Benchluch S. M. Et al. 1993. A Trayectory Sensitivity Method for the Identification of Nonlinear Excitation System Models. IEEE Transactions on Energy Conversion, Jun 1993.

[2] Estudios Electricos SRL. 2003. Estudio Técnico sobre Ensayos y Ajustes en el Complejo de Ecopetrol-Barrancabermeja. 2003

[3] Feltes J. W. Et al. 2002. Deriving Model Parameters from Field Test Measurements. IEEE Computer Applications in Power, Oct 2002.

[4] Gibbard M. J. et al. 1975. Identification of Excitation System Parameters. IEEE Transactions on Power Apparatus and Systems, Jul/Ago 1975 .

[5] Guo T. Y. Et al. 1995. Identification of Model Parameters of Excitation Systems and Power System Satabilizer of Mingtan 6 via Finalization Fiels Test. IEEE Transactions on Power Systems, May 1995.

[6] IEEE Std 421.5. 1992. Recommended Practice for Excitation System Models for Power System Stability Studies.

[7] Liaw C. M. Et al. 1992. Parameter Estimation of Excitation Systems from Sampled Data. IEEE Transactions on Automatic Control, May 1992.

[8] Ljung, L. 1987. System Identification: Theory for the User. Prentice Hall 1987

[9] Ludwig E. Et al. 1998. A Feasibility Study of On Line Excitation System Parameters Estimation. IEEE Transactions on Power Systems, Ago 1998

[10] Orozco, Martha. 2004. Diseño e Implementación de un Regulador de Voltaje para un Generador Sincrónico. Reporte Interno Posgrado en Ingeniería - Automática. Universidad del Valle.

[11] Ramírez, J. M. Et al. 1999. Modelos Matemáticos para los Reguladores de Velocidad y los Sistemas de Excitación de la Planta de Salvajina. Contrato No MAN-078-99 Universidad del Valle-EPSA. Dic 1999.

[12] Ramírez, J. M. Et al. 2000. Modelos Matemáticos para los Reguladores de Velocidad y los Sistemas de Excitación de la Planta de Salvajina. Energía y Computación. No 2, 2000

[13] Saavedra, A. J. 2002. Modelado Para Estudios de Estabilidad de los Sistemas de Control Velocidad y Excitación de la Central de Salvajina. Tesis de Maestría, Universidad del Valle, 2002.

[14] Sanchez J. J. et al. 1988. Trayectory Sensitivity Based Identification of Synchronous Generator and Excitation Systems Parameters. IEEE Transactions on Power Systems, Nov 1988.

[15] Soderstrom, Torsten. 1989. System Identification. Prentice Hall. 1989.

[16] Wang J. C. Et al. 1995. Identification of Excitation Systems Models Based on Line Digital Measurements. IEEE Transactions on Power Systems, Ago 1995 